\title{
Anti-ubiquitin immunocytochemistry is more sensitive than conventional techniques in the detection of diffuse Lewy body disease
}

\author{
GRAHAM LENNOX, ${ }^{*} \dagger$ JAMES LOWE, $\dagger$ KEN MORRELL, $\dagger$ MICHAEL LANDON, \\ R JOHN MAYER‡
}

From the Departments of Neurology,* Neuropathology, $\dagger$ and Biochemistry, $\ddagger$ Queen's Medical Centre, Nottingham, $U K$

SUMMARY Brainstem and cortical Lewy bodies in diffuse Lewy body disease show intense immunoreactivity to antibodies against ubiquitin. Quantitative studies show that the novel neuropathological technique of anti-ubiquitin immunocytochemistry is more than twice as sensitive as conventional haematoxylin and eosin stains in detecting cortical Lewy bodies. Anti-ubiquitin immunocytochemistry should be regarded as the method of choice for the diagnosis and quantificaton of diffuse Lewy body disease.

Diffuse Lewy body disease is characterised pathologically by the presence of rounded eosinophilic intraneuronal inclusion bodies (Lewy bodies) in brainstem nuclei (where the changes are indistinguishable from those of idiopathic Parkinson's disease) and throughout the cerebral cortex. ${ }^{1}$ The disease has recently attracted attention as a potential pathological substrate of dementia in Parkinson's disease, ${ }^{23}$ but studies of diffuse Lewy body disease are hampered by the difficulty of detecting cortical Lewy bodies using conventional staining techniques. Whilst brainstem Lewy bodies are intensely eosinophilic, are often surrounded by a pale halo, and are in any case highlighted by surrounding neuromelanin, their corticol counterparts are by contrast less eosinophilic and less clearly circumscribed (fig 1). This makes it difficult to diagnose cases of diffuse Lewy body disease using conventional haematoxylin and eosin (H \& E) stains.

The traditional approach to this problem is the use of alternative stains for Lewy bodies like Lendrum's phloxine tartrazine; such stains are, however, unreliable in demonstrating cortical Lewy bodies and add little to the sensitivity of conventional $H \& E$ stains. Silver staining methods are also apparently unreliable, with reports of both positive ${ }^{14}$ and negative $^{56}$ silver staining of cortical Lewy bodies.

Address for reprint requests: Dr Graham Lennox, Department of Neurology, Queen's Medical Centre, Nottingham, NG7 2UH, UK.

Received 17 May 1988 and in revised form 18 September 1988. Accepted 22 September 1988
More recently it has been established that Lewy bodies can be stained immunocytochemically using antibodies directed against cytoskeletal elements such as neurofilaments. ${ }^{6-8}$ However, a large proportion of cortical Lewy bodies do not show immunoreactivity with these techniques (unpublished observations), limiting their usefulness in both diagnosing and quantifying diffuse Lewy body disease.

It has recently been shown that Lewy bodies can be stained immunocytochemically using antibodies directed against ubiquitin. ${ }^{910}$ The present study aims to determine the sensitivity of this technique in the detection of cortical Lewy bodies, and thus in the diagnosis and quantification of diffuse Lewy body disease.

\section{Materials and methods}

These studies were performed on ten of the 15 cases of diffuse Lewy body disease identified by the presence of both cortical and classical brainstem Lewy bodies in a survey of the 216 brains referred to this institution from within the Nottingham Health District during a single year. The clinical details of these cases will be reported elsewhere. All brains had been fixed in $10 \%$ formol saline for at least three months (range 336 months). Blocks from the anterior cingulate gyrus were then processed in paraffin wax; this region was selected because preliminary studies demonstrated that in general it is in this region that cortical Lewy bodies are most easily recognised using $H$ \& $E$ stains. Serial sections cut at 10 micrometres were coded and stained using conventional $\mathbf{H}$ \& $E$ and anti-ubiquitin immunocytochemistry.

The production and specificity of the anti-ubiquitin antibody has been described in detail elsewhere. 9 "In brief, it 


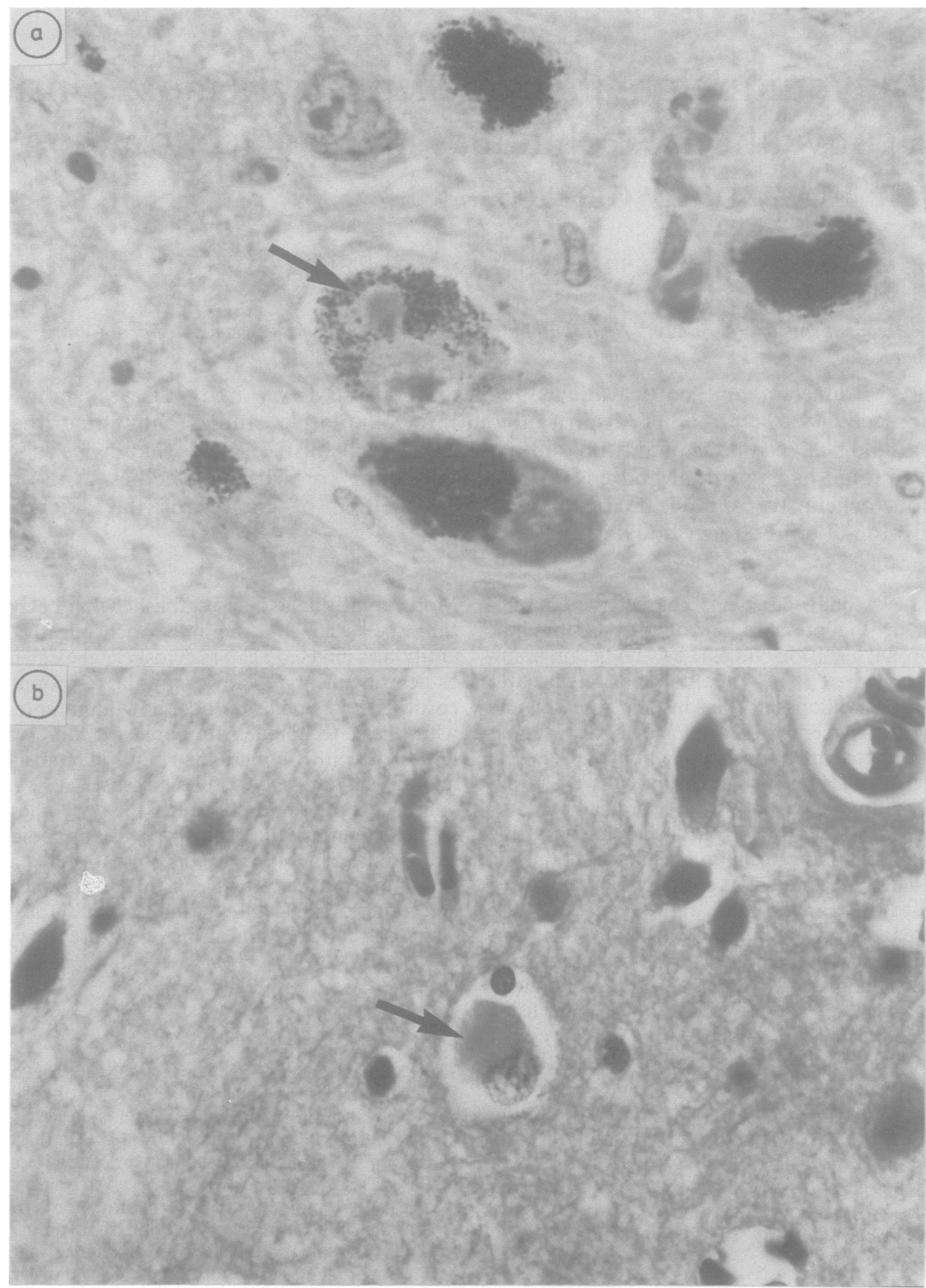

Fig 1 Neurons containing Lewy bodies in ( $a$ ) the substantia nigra and (b) anterior cingulate gyrus in a case of diffuse Lewy body disease stained with $H \& E$. Original magnification $\times 950$. 
was produced by immunising rabbits with purified calf thymus ubiquitin conjugated to bovine gamma-globulin. The resulting polyclonal IgG antibody was purified by ammonium sulphate precipitation and DEAE-cellulose chromatography. It showed high specificity for ubiquitin-protein conjugates (such as ubiquitin-bovine-gamma-globulin, ubiquitin-haemocyanin and ubiquitin-histone $\mathrm{H} 2 \mathrm{~A}$ ) as assessed both by ELISA and SDS-PAGE immunoblotting techniques and by absorption controls.

The anti-ubiquitin antibody was used (at a dilution of 1:500) to stain tissue sections using standard indirect peroxidase and streptavidin biotin techniques. ${ }^{12}$ Both brainstem and corticol Lewy bodies showed intense immunoreactivity which was generally most intense peripherally (that is, in that part of the Lewy body thought to contain intact neurofilament epitopes') (fig 2). Serial sections and stainrestain studies showed that the ubiquitin immunoreactivity was localised to classical Lewy bodies, to similar but less clearly circumscribed intraneuronal hyaline eosinophilic inclusion bodies (so-called Lewy-like bodies) and to the noneosinophilic intraneuronal hyaline or slightly granular inclusion bodies seen in diffuse Lewy body disease (so-called pale bodies') (fig 3). For quantitative studies using H \& E stains both classical Lewy bodies and Lewy-like bodies were counted, but pale bodies were not because they are extremely difficult to identify unequivocally. Using anti-ubiquitin immunocytochemistry Lewy bodies were defined as rounded, non-fibrillary and densely immunoreactive inclusion bodies; preliminary studies established that this definition distinguished between Lewy bodies and other inclusion bodies such as globose neurofibrillary tangles, Pick bodies etc, but included pale bodies.

Quantitative studies were performed by marking the area to be assessed in ink on the slide coverslips, using anatomical landmarks such as sulci and blood vessels to ensure that an identical area was studied in both the $\mathrm{H} \& \mathrm{E}$ and antiubiquitin immunocytochemistry slides. This area was then systematically scanned by an experienced observer using an eyepiece graticule at a magnification of $\times 250$ and numbers of Lewy bodies were recorded. H \& $E$ and anti-ubiquitin immunocytochemistry slides were studied in random order on different days, and both were reasssessed by the same observer three months later, without reference to previous counts.

To make certain that identical areas had indeed been assessed by the two techniques the areas of study were then projected using a calibrated Nikon Slide Projector, traced and quantified using a Kontron Videoplan Computerised Planimeter. The mean area assessed per slide was $78.7 \mathrm{~mm}^{2}$ (range 39.9-114.9 $\mathrm{mm}^{2}$ ) and in no case did the area of the $\mathrm{H}$ \& E slide differ from its anti-ubiquitin immunocytochemistry counterpart by more than $2 \%$.

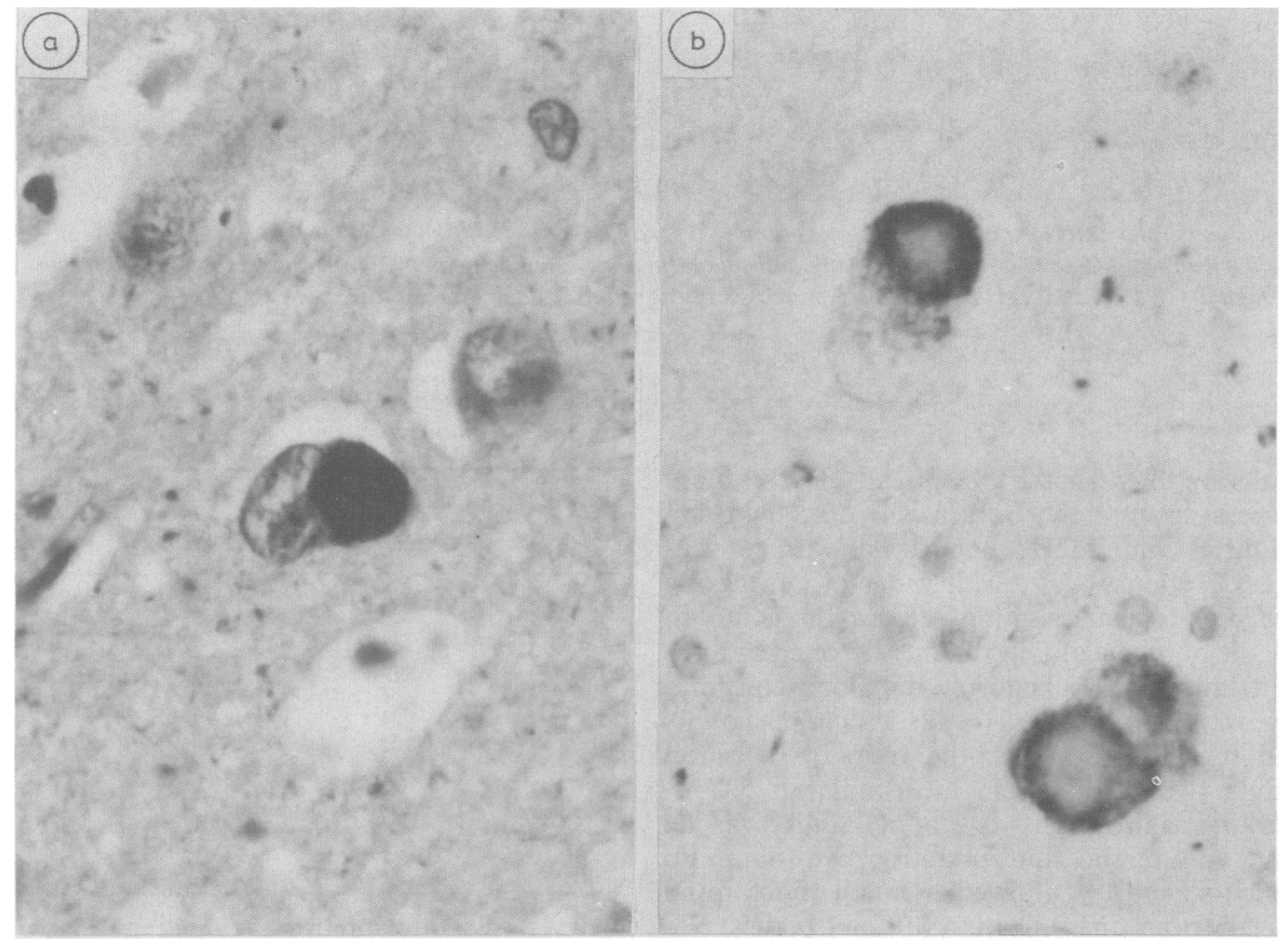

Fig 2 Neurons containing Lewy bodies in the anterior cingulate gyrus in the same case of diffuse Lewy body disease stained immunocytochemically with anti-ubiquitin antibody $(a)$ at the dilution used throughout this study (1:500), and (b) at greater dilution in order to illustrate the more intense peripheral staining.

Magnification $\times 950$. 


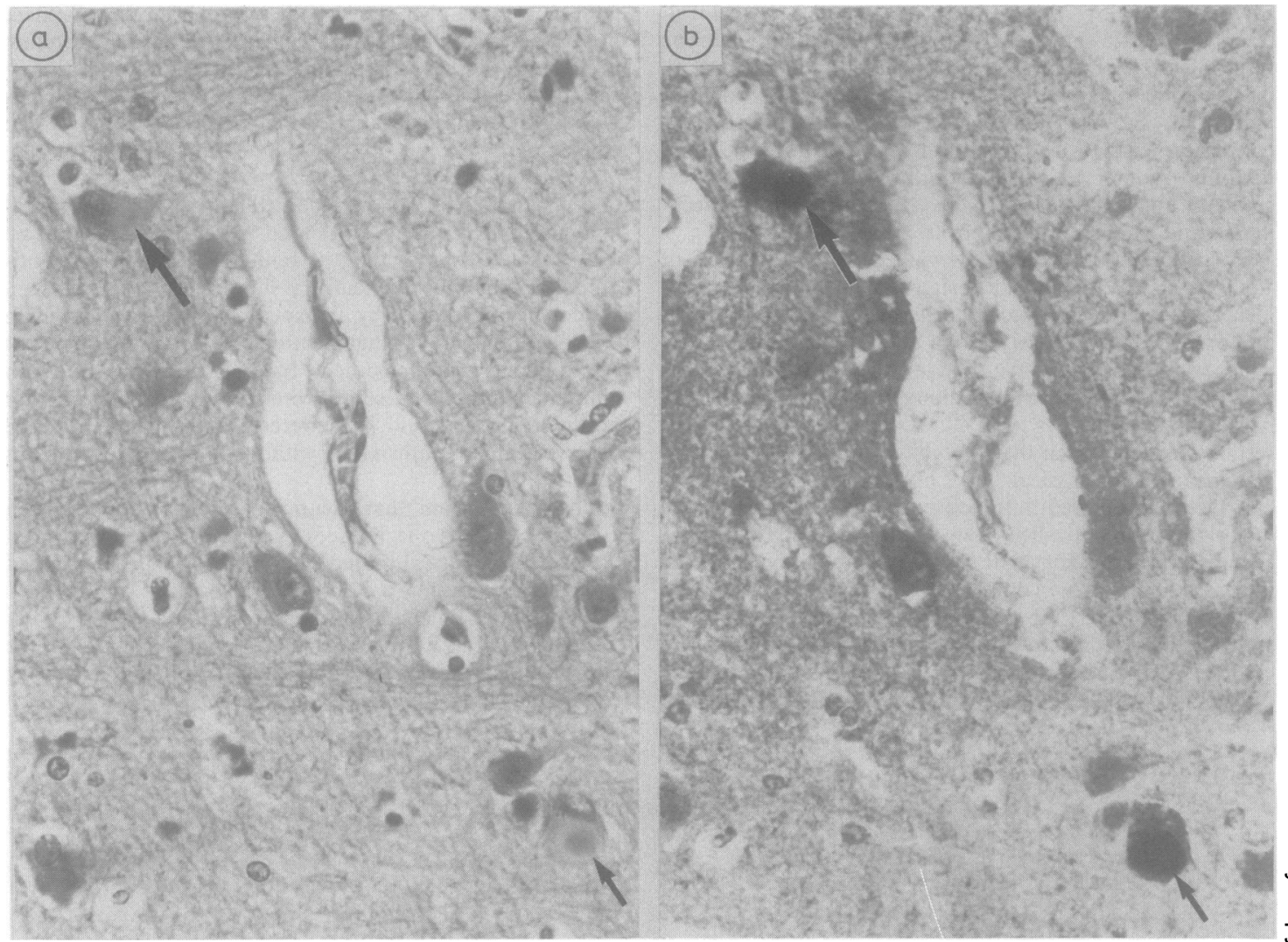

Fig 3 Neurons in the anterior cingulate gyrus containing a cortical Lewy body (short arrow) and a pale body (long arrow) (a) stained with $H$ and $E$ and (b) restained using anti-ubiquitin immunocytochemistry: both inclusions show intense immunoreactivity. (Note that restaining causes increased background staining.) Magnification $\times 560$.

\section{Results}

The results are shown in the table. In all ten cases more than twice as many Lewy bodies were detected using anti-ubiquitin immunocytochemistry as with conventional H \& E stains, over a wide range of Lewy body densities. On repeat counting after a three month interval, the numbers of Lewy bodies detected by antiubiquitin immunocytochemistry remained constant, but there were discrepancies $(+/-5 \%)$ in the counts obtained by $\mathbf{H} \&$ E stains; the ratio of detection between the two methods remained similar at 2.27.

As striking as this sensitivity and reliability was the speed and ease of slide interpretation; anti-ubiquitin immunocytochemistry allowed a much more rapid and less laborious quantification of Lewy bodies.

\section{Discussion}

This study confirms the previous observations that
Table Number of Lewy bodies detected by $H \& E$ and antiubiquitin immunocytochemistry

\begin{tabular}{lccc}
\hline \multirow{4}{*}{ Case number } & \multicolumn{3}{l}{ Number of Lewy bodies detected } \\
\cline { 2 - 4 } & $H$ and $E$ & Anti-ubiquitin & Ratio \\
\hline 1 & 13 & 32 & $2 \cdot 41$ \\
2 & 8 & 18 & $2 \cdot 25$ \\
3 & 15 & 36 & $2 \cdot 40$ \\
4 & 15 & 34 & $2 \cdot 27$ \\
5 & 58 & 121 & $2 \cdot 08$ \\
6 & 21 & 48 & $2 \cdot 29$ \\
7 & 153 & 378 & $2 \cdot 47$ \\
8 & 24 & 61 & $2 \cdot 54$ \\
9 & 53 & 119 & $2 \cdot 25$ \\
10 & 127 & 319 & $2 \cdot 51$ \\
\hline & & & Mean: $2 \cdot 35$ \\
\hline
\end{tabular}

Lewy bodies are intensely stained by anti-ubiquitin immunocytochemistry. Ubiquitin is, as its name suggests, found in abundance throughout the natural world. It is a small and highly conserved protein which is produced in cells in response to a variety of 
experimental stresses including experimental heat shock. ${ }^{13}$ Although its functions are not fully understood, ubiquitin is known to have a major role in mediating extralysosomal proteolysis; it conjugates with ("ubiquitinates") short-lived and abnormal proteins, thus targeting them for cytosolic degradation. ${ }^{14}$ Its presence within Lewy bodies has not yet been explained, although it is tempting to speculate that it reflects an unsuccessful attempt to degrade an abnormal protein within the cell. ${ }^{9}$

There are a number of possible explanations for the superiority of anti-ubiquitin immunocytochemistry over conventional stains in the detection of cortical Lewy bodies. Firstly, unlike for example the silver stains for neurofibrillary tangles, there has in the past been no reliable "special stain" for the Lewy body. H \& E stains provide poor contrast between cortical Lewy bodies and surrounding cytoplasm, making it difficult to identify small (or tangentially sectioned) Lewy bodies, whereas the intensity of staining achieved with anti-ubiquitin immunocytochemistry makes this easy. Secondly, H \& E stains fail to stain pale bodies which are intensely stained using antiubiquitin immunocytochemistry, suggesting that they should be regarded as part of the pathology of diffuse Lewy body disease. Thirdly, it is possible that antiubiquitin immunocytochemistry is more sensitive than other immunocytochemical techniques because the ubiquitin epitopes within the Lewy body are more accessible to antibody (whether because ubiquitin is a smaller molecule, present in larger amounts, or located more superficially) than other epitopes such as those of neurofilament. Fourthly, it is possible, although not proven by this study, that anti-ubiquitin immunocytochemistry is revealing a subset of inclusion bodies which are not visualised using conventional stains. This is certainly so when the technique is applied to cases of motor neuron disease, where anti-ubiquitin immunocytochemistry reveals inclusion bodies in spinal anterior horn cells which at present cannot be detected using any other technique."

This study also demonstrates the improvement in intraobserver reliability that is obtained with the more sensitive technique. Lewy body counts varied significantly on recounting $\mathrm{H} \& \mathrm{E}$ sections but remained constant on recounting with anti-ubiquitin immunocytochemistry, even though the quantification of the latter was much more rapid. Furthermore, antiubiquitin immunocytochemistry allows the detection of Lewy bodies (and the related pale bodies) with high specificity. Lewy bodies are, for example, readily distinguished from globose neurofibrillary tangles by their non-fibrillary architecture and by the fact that whilst perikaryal they do not envelope the nucleus, and from Pick bodies by their dense and homogeneous immunoreactivity.

These observations have practical implications in that anti-ubiquitin immunocytochemistry should now be regarded as the method of choice for the diagnosis and quantification of diffuse Lewy body disease. They also have theoretical implications, since the finding that Lewy bodies contain ubiquitin and can be sensitively stained by the anti-ubiquitin immunocytochemistry technique opens up the intriguing possibility of isolating the Lewy body, determining its biochemical basis and thus obtaining an understanding of the molecular pathology of Parkinson's disease.

These studies were supported by The Stanhope Trust and The Parkinson's Disease Society. The authors are grateful to Dr R B Godwin-Austen for advice and support, to Mr D McQuire for technical assistance and to $\mathrm{Mr} \mathrm{W}$ Brackenbury for photomicrography.

\section{References}

1 Gibb WRG, Esiri MM, Lees AJ. Clinical and pathological features of diffuse cortical Lewy body disease (Lewy body dementia). Brain 1987;110:1131-53.

2 Byrne EJ, Lowe J, Godwin-Austen RB, et al. Dementia and Parkinson's disease associated with diffuse cortical Lewy bodies. Lancet 1987; i:501.

3 Lennox G, Lowe JS, Byrne J, et al. Cortical Lewy bodies and the spectrum of Lewy body disease. J Neurol Neurosurg Psychiatry 1988;51:135-8.

4 Kosaka K. Lewy bodies in cerebral cortex. Report of three cases. Acta Neuropathol (Berl.) 1978;42:127-34.

5 Forno LS. The Lewy body in Parkinson's disease. $A d v$ Neurol 1986;45:35-42.

6 Dickson DW, Davies P, Mayeux R, et al. Diffuse Lewy body disease. Neuropathological and biochemical studies of six patients. Acta Neuropathol. (Berl.) 1987;75:8-15.

7 Goldman JE, Yen S-H, Chiu F-C, et al. Lewy bodies of Parkinson's disease contain neurofilament antigens. Science 1983;221:1082-4.

8 Forno LS, Sternberger LA, Sternberger NH, et al. Reaction of Lewy bodies with antibodies to phosphorylated and non-phosphorylated neurofilaments. Neurosci Lett 1986;64:253-8.

9 Lowe J, Blanchard A, Morrell $\mathrm{K}$, et al. Ubiquitin is a common factor in intermediate filament inclusion bodies of diverse type in man, including those of Parkinson's disease, Pick's disease and Alzheimer's disease, as well as Rosenthal fibres in cerebellar astrocytomas, cytoplasmic bodies in muscle and Mallory bodies in alcoholic liver disease. $J$ Pathol 1988;155:9-15.

10 Kuzuhara S, Mori H, Izumiyama N, et al. Lewy bodies are ubiquitinated: a light and electron microscopic immunocytochemical study. Acta Neuropathol (Berl.) 1988;75:345-53.

11 Lowe J, Lennox G, Jefferson D, et al. A filamentous inclusion body within anterior horn neurones in motor neurone disease defined by immunocytochemical localisation of ubiquitin. Neurosci Lett (in press).

12 Sternberger LA. Immunocytochemistry, 3rd ed. New York: Wiley, 1986.

13 Finley D, Varshavsky A. The ubiquitin system: functions and mechanisms. Trends in Biochem Sci 1985;10:343-7.

14 Rechsteiner M. Ibiquitin mediated pathways for intracellular proteolysis. Annu Rev Cell Biol 1987;3: 1-30. 